\title{
Gravity Binding and Pressure Bounding of HII regions and Molecular Clouds in Interacting Galaxies
}

\author{
J. Zaragoza-Cardiel ${ }^{1}$, J. Beckman ${ }^{2,3,4}$, J. Font ${ }^{2,3}$, A. Camps-Fariña ${ }^{2,3}$, \\ B. Garcìa-Lorenzo ${ }^{2,3}$ and A. Serrano-Borlaff ${ }^{2,3}$ \\ ${ }^{1}$ Instituto de Astronomía, Universidad Nacional Autónoma de México, Mexico City, Mexico \\ email: javier.zaragoza@astro.unam.mx \\ ${ }^{2}$ Instituto de Astrofísica de Canarias, C/ Vía Láctea s/n, 38205 La Laguna, Tenerife, Spain \\ ${ }^{3}$ Department of Astrophysics, University of La Laguna, E-38200 La Laguna, Tenerife, Spain \\ ${ }^{4}$ CSIC, 28006 Madrid, Spain
}

\begin{abstract}
We have observed 3 pairs of interacting galaxies (the Antennae, Arp 236, and NGC 1614) using the Fabry-Perot interferometer GH $\alpha$ FaS (Galaxy H $\alpha$ Fabry-Perot system) on the 4.2m William Herschel Telescope (WHT) at the Observatorio del Roque de los Muchachos, La Palma. We have extracted the physical properties (sizes, $\mathrm{H} \alpha$ luminosity and velocity dispersion) of HII regions. We have combined also these observations with ALMA archival observations of these interacting galaxies, finding that there is a set of brighter and denser star forming regions. We have been able to compare these properties with those of two SMGs at redshift $\sim 2$.
\end{abstract}

Keywords. Interacting galaxies, star formation, HiI regions, GMCs.

\section{Introduction}

Galaxy mergers produce massive galaxies in hierarchical models of galaxy formation, however there is some evidence that these models are incorrect, or at best incomplete. The process of star formation enhancement in interacting galaxies is postulated but not very well understood. The study of the kinematics of the star forming regions in galaxy mergers turns out to be crucial to clarify if star formation is indeed enhanced, how the extra-nuclear star forming regions are caused, and to characterize the physical parameters of the gas in these regions. Using ASTRODENDRO (Rosolowsky et al. 2008) Zaragoza-Cardiel et al. (2015) were able to measure the radius $(R)$, the velocity dispersion $\left(\sigma_{v}\right)$ and the $\mathrm{H} \alpha$ luminosity $\left(L_{\mathrm{H} \alpha}\right)$ for each HiI region observed in 8 pairs of interacting galaxies. The data used in Zaragoza-Cardiel et al. (2015) is already publicly available.1

In Zaragoza-Cardiel et al. (2015) we show that there is a population of Hir regions in interacting galaxies which is brighter, denser, and more turbulent than the regions in isolated galaxies. This demonstrates quantitatively that star formation and the turbulence is enhanced in the sample of interacting galaxies compared with the sample of isolated galaxies.

\section{HII regions and GMCs in interacting galaxies}

We have observed the Antennae galaxies, Arp 236, and NGC 1614 with GH $\alpha \mathrm{FaS}$ and combined these observations with public ALMA data. Using ASTRODENDRO we have extracted the properties of HII regions and GMCs in those galaxies. The virial parameter decreases with the mass of the star forming regions (Fig. 1 top), which implies that the more massive the region, the more important is the gravity rather than the external pressure (Bertoldi \& McKee 1992).. We find also that the gas surface density and the star formation rate surface density is enhanced for the massive star forming regions (Fig. 1 bottom). The properties of the massive star forming 

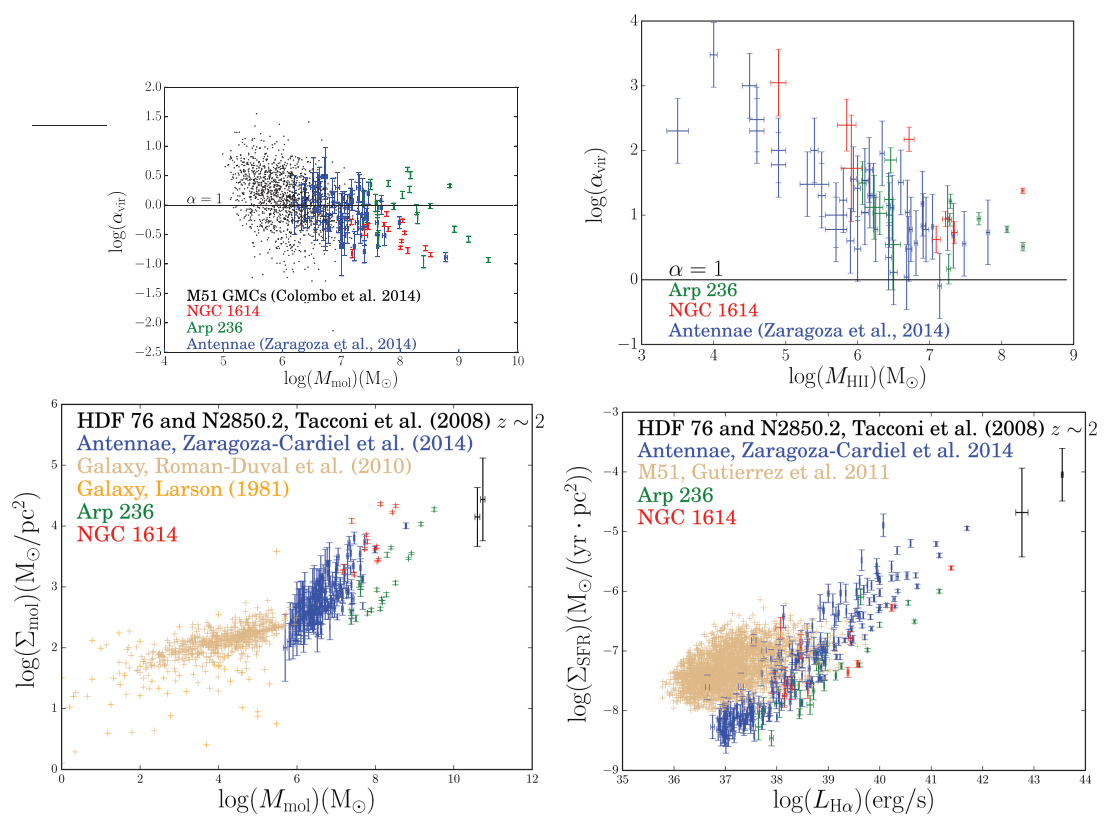

Figure 1. Top left: Virial parameter, $\alpha_{\mathrm{vir}}$, versus molecular gas mass, $M_{\mathrm{mol}}$, of GMCs. Top right: $\alpha_{\mathrm{vir}}$ versus ionized gas mass, $M_{\mathrm{HII}}$, of HII regions. Bottom left: Gas surface density, $\Sigma_{\mathrm{mol}}$, versus $M_{\mathrm{mol}}$ of GMCs. Bottom right: Star formation rate surface density, $\Sigma_{\mathrm{SFR}}$, versus $M_{\mathrm{HI}}$ of HiI regions.

regions found in these interacting galaxies, connect the properties of the star forming regions in the Milky Way and M51, with the properties of two extreme star forming galaxies (HDF 76 and $\mathrm{N} 2850.2$ ) at redshift $\sim 2$.

\section{Conclusions}

We found a population of massive star forming regions where the star formation is enhanced compared with the properties of star forming regions in non interacting galaxies. This enhancement could explain the extreme star formation at higher redshifts.

\section{References}

Bertoldi, F., \& McKee, C. F. 1992, ApJ, 395, 140

Colombo, D., Hughes, A., Schinnerer, E., et al. 2014, ApJ, 784, 3

Gutiérrez, L., Beckman, J. E., \& Buenrostro, V. 2011, AJ, 141, 113

Larson, R. B. 1981, MNRAS, 194, 809

Roman-Duval, J., Jackson, J. M., Heyer, M., Rathborne, J., \& Simon, R. 2010, ApJ, 723, 492

Rosolowsky, E. W., Pineda, J. E., Kauffmann, J., \& Goodman, A. A. 2008, ApJ, 679, 1338

Tacconi, L. J., Genzel, R., Smail, I., et al. 2008, ApJ, 680, 246

Zaragoza-Cardiel, J., Font, J., Beckman, J. E., et al. 2014, MNRAS, 445, 1412

Zaragoza-Cardiel, J., Beckman, J. E., Font, J., et al. 2015, MNRAS, 451, 1307 